EPJ Web of Conferences 69, 00003 (2014)

DOI: $10.1051 /$ epjconf / 20146900003

(C) Owned by the authors, published by EDP Sciences, 2014

\title{
Toward a self-consistent and unitary reaction network for big- bang nucleosynthesis
}

\author{
Mark W. Paris ${ }^{1, a}$, Lowell S. Brown ${ }^{1}$, Gerald M. Hale ${ }^{1}$, Anna C. Hayes-Sterbenz ${ }^{1}$, Gerard \\ Jungman ${ }^{1}$, Toshihiko Kawano ${ }^{1}$, George M. Fuller ${ }^{2}$, Evan B. Grohs ${ }^{2}$, and Satoshi Kunieda ${ }^{3}$ \\ ${ }^{1}$ Los Alamos National Laboratory, Los Alamos, New Mexico 87545, USA \\ ${ }^{2}$ Department of Physics, University of California, San Diego, La Jolla, California 92093, USA \\ ${ }^{3}$ Nuclear Data Center, Japan Atomic Energy Agency, Tokai-mura Naka-gun, Ibaraki 319-1195, Japan
}

\begin{abstract}
Unitarity, the mathematical expression of the conservation of probability in multichannel reactions, is an essential ingredient in the development of accurate nuclear reaction networks appropriate for nucleosynthesis in a variety of environments. We describe our ongoing program to develop a "unitary reaction network" for the big-bang nucleosynthesis environment and look at an example of the need and power of unitary parametrizations of nuclear scattering and reaction data. Recent attention has been focused on the possible role of the ${ }^{9} \mathrm{~B}$ compound nuclear system in the resonant destruction of ${ }^{7} \mathrm{Li}$ during primordial nucleosynthesis. We have studied reactions in the ${ }^{9} \mathrm{~B}$ compound system with a multichannel, two-body unitary R-matrix code (EDA) using the known elastic and reaction data, in a four-channel treatment. The data include elastic ${ }^{6} \mathrm{Li}\left({ }^{3} \mathrm{He},{ }^{3} \mathrm{He}\right){ }^{6} \mathrm{Li}$ differential cross sections from 0.7 to $2.0 \mathrm{MeV}$, integrated reaction cross sections for energies from 0.7 to $5.0 \mathrm{MeV}$ for ${ }^{6} \mathrm{Li}\left({ }^{3} \mathrm{He}, p\right)^{8} \mathrm{Be}^{*}$ and from 0.4 to $5.0 \mathrm{MeV}$ for the ${ }^{6} \mathrm{Li}\left({ }^{3} \mathrm{He}, d\right){ }^{7} \mathrm{Be}$ reaction. Capture data have been added to the previous analysis with integrated cross section measurements from 0.7 to $0.825 \mathrm{MeV}$ for ${ }^{6} \mathrm{Li}\left({ }^{3} \mathrm{He}, \gamma\right){ }^{9} \mathrm{~B}$. The resulting resonance parameters are compared with tabulated values from TUNL Nuclear Data Group analyses. Previously unidentified resonances are noted and the relevance of this analysis and a unitary reaction network for big-bang nucleosynthesis are emphasized.
\end{abstract}

\section{INTRODUCTION}

The light nuclear reaction program, anchored at Los Alamos National Laboratory and bringing together researchers from various disciplines, employs a dual approach in gaining a detailed understanding of the structure and reactions of light nuclei $(A \lesssim 20)$. This work is accomplished in the service of end-users in both applied and fundamental disciplines such as nuclear security and nuclear astrophysics. We apply dual, complementary methods of phenomenological ( $R$ matrix) and $a b$ initio (quantum Monte Carlo - QMC) techniques to constrain and describe data sets of light nuclear scattering and reaction observables.

Motivations for revisiting the status and consistency of nuclear reaction networks in big-bang nucleosynthesis $(\mathrm{BBN})$ are manifold. Beyond addressing whether nuclear physics can further constrain

\footnotetext{
ae-mail: mparis@lanl.gov
} 
the "lithium problem" discussed below, we are interested in developing BBN as a tool for precision cosmology. A primary objective in this endeavor is to implement the 'theoretical minimum' of incorporating the conservation of probability - unitarity - into the nuclear reaction network. This objective has yet to be attempted in a comprehensive fashion. In the traditional approach, when the observed integrated cross section is available, it is fit with a smooth curve and transformed to a Maxwellianaveraged reactivity, proportional to $\langle\sigma v\rangle$. We have found that cross sections determined in this way (and by less well established methods, such as the narrow resonance approximation) can lead to incorrect normalizations by significant factors. In addition to establishing consistent, comprehensive parametrizations of the nuclear reaction network, we are motivated to revisit BBN as a tool of precision cosmology as new observational techniques of primordial elements (for example, recent observation of primordial deuterium abundance[1]) have achieved a precision at the two-percent level. At the same time, the Planck mission[2] and new 30m-class of radio telescopes[3] will challenge existing methods of primordial abundance calculations, which are based largely on the original methods of Wagoner[4]. A parallel objective in our current work is to couple the unitary reaction network (URN) to a full Boltzmann neutrino transport code. Such a code would be useful, we think, to the community to test such theories as active-sterile $v$ mixing and massive particle out-of-equilibrium decays associated with beyond-standard model unstable particles[5].

We are mainly concerned in this presentation with the phenomenological $R$ matrix approach, we stress the importance and essential character of unitary parametrization of the compound nuclear system corresponding to multichannel scattering and reaction observables. This parametrized approach to gaining a simultaneous understanding of multichannel data, such as integrated and differential cross section $\left(\sigma(E)\right.$ and $\frac{d \sigma}{d \Omega}$, respectively) and polarization observables $\left(A_{i}(\theta), C_{i, j}, K_{i}^{j^{\prime}}, \Sigma(\gamma), \ldots\right)$ allows the extraction of the elements of the transition matrix $T_{f i}$ for the process channel $i \rightarrow$ channel $f$. Given an explicit expression for the $T$ matrix, we have a complete description of the compound nuclear system - including for example its resonance structure. As a constraint, unitarity is highly restrictive and may be used to determine the consistency of data sets within and across experiments, as we will show in Sec.2.1.

After we review the $R$ matrix formalism, we consider the impact of several analyses of compound nuclear systems. As a specific instance of the impact of our work on the field of nuclear data, we will consider the case of the ${ }^{17} \mathrm{O}$ compound system, which has been analyzed by Hale[6] via the $R$ matrix code EDA. We demonstrate that unitarity provides a constraint on the absolute and relative scales of the normalizations of different experiments' measurements of the integrated cross section. A example of

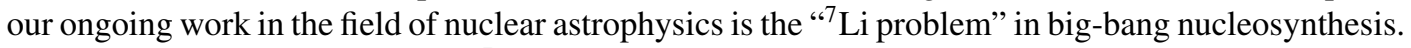
Calculations of the abundance of ${ }^{7} \mathrm{Li}[7]$ overestimate the value extracted from observations of lowmetallicity halo dwarf stars[8], where the stellar dynamics are supposed to be sufficiently understood to isolate the primordial ${ }^{7} \mathrm{Li}$ component. The discrepancy with this (and another[9]) observation by a factor of $2.2 \leftrightarrow 4.2$ corresponds to a deviation of $4.5 \sigma \leftrightarrow 5.5 \sigma$, a result that has only become more severe with time. It is essential to determine the nature of this discrepancy as BBN probes conditions of the very early universe and our understanding of physical laws relevant in an extreme environment.

Recent attention has focused on the role of reactions that destroy $A=7$ nuclei at early times $\lesssim 1 \mathrm{~s}$ in the big-bang environment[10, 11]. The authors of Ref.[10], citing the TUNL-Nuclear Data Group (NDG) evaluation tables[12], (See Table 1.) conjecture that the putative $5 / 2^{+}$resonance near 16.7 $\mathrm{MeV}$ may enhance the destruction of ${ }^{7} \mathrm{Be}$ through reactions such as ${ }^{7} \mathrm{Be}(\mathrm{d}, \mathrm{p}) \alpha \alpha$ and ${ }^{7} \mathrm{Be}(\mathrm{d}, \gamma){ }^{9} \mathrm{~B}$ if the resonance parameters are within given ranges. These studies employ the Wigner limit for the reduced width[13] to determine an upper bound on the contribution of resonances, particularly ${ }^{9} \mathrm{~B}$, to a resonant enhancement in reactions that destroy mass-7 nuclides, ${ }^{7} \mathrm{Li}$ in particular. Because there is a paucity of data in the region near the $\mathrm{d}-{ }^{7} \mathrm{Be}$ threshold which the assumed $\frac{5}{2}{ }^{+} \mathrm{B}$ resonance inhabits, 
Table 1. The TUNL-NDG/ENSDF resonances in the ${ }^{9} \mathrm{~B}$ compound nuclear system[12] near the $\mathrm{d}-{ }^{7} \mathrm{Be}$ threshold at $16.49 \mathrm{MeV}$.

\begin{tabular}{|c|c|c|c|}
\hline$E_{x}(\mathrm{MeV} \pm \mathrm{keV})$ & $J^{\pi} ; T$ & $\Gamma_{\mathrm{cm}}(\mathrm{keV})$ & Decay \\
\hline $16.024 \pm 25$ & $T=\left(\frac{1}{2}\right)$ & $180 \pm 16$ & \\
$16.71 \pm 100$ & $\left(\frac{5}{2}^{+}\right) ;\left(\frac{1}{2}\right)$ & & \\
$17.076 \pm 4$ & $\frac{1}{2}^{-} ; \frac{3}{2}^{2}$ & $22 \pm 5$ & $\left(\gamma,{ }^{3} \mathrm{He}\right)$ \\
$17.190 \pm 25$ & & $120 \pm 40$ & $\mathrm{p}, \mathrm{d},{ }^{3} \mathrm{He}$ \\
$17.54 \pm 100$ & $\left(\frac{7}{2}^{+}\right) ;\left(\frac{1}{2}\right)$ & & \\
$17.637 \pm 10$ & & $71 \pm 8$ & $\mathrm{p}, \mathrm{d},{ }^{3} \mathrm{He}, \alpha$ \\
\hline
\end{tabular}

we wondered if the existing data may indicate the presence of such a resonance if a multichannel, unitary $R$-matrix evaluation is pursued.

Our motivation for the present study of the ${ }^{9} \mathrm{~B}$ compound system is two-fold. The continuing light nuclear reaction program at Los Alamos National Laboratory, T-2 Theoretical Division provides light nuclear data for an array of end users, including the ENDF and ENSDF communities. Moreover, we are interested in updating the evaluation of the ${ }^{9} \mathrm{~B}$ compound system to address the key question outlined above for BBN: does a resonance near the $\mathrm{d}-{ }^{7} \mathrm{Be}$ threshold cause an increase in the destruction of mass-7 nuclides in the early universe and possibly explain the ${ }^{7} \mathrm{Li}$ overprediction problem?

We consider in the next section, Sec.4, some work on the effective field theory (EFT) description of light nuclei. Here, we study a simple model EFT of interacting neutrons, deuterons, tritons, helions, and an ${ }^{5} \mathrm{He}$ * degree-of-freedom corresponding to the compound nuclear system relevant to the $d t \rightarrow$ $n \alpha$ reaction, which is of great importance to fusion processes driven by fusion. We compare the EFT to the zero-channel radii limit of a two-channel $R$ matrix approach and demonstrate the interesting fact that these different descriptions are identical. A fit of the observed data of high fidelity is obtained in these approaches with three parameters.

\section{The $R$-matrix approach}

The $R$-matrix approach[14-16] is a unitary, multichannel parametrization that has proven useful for an array of nuclear reaction phenomenology, particularly for light nuclei[17]. We give only a brief description here and refer to the literature for a more complete description[18, 19].

We consider formal unitarity and the $R$ matrix formalism in this section and apply it to the analysis of the compound nuclear systems: ${ }^{9} \mathrm{~B},{ }^{13} \mathrm{C},{ }^{14} \mathrm{C}$ and ${ }^{17} \mathrm{O}$.

\subsection{Unitarity as a constraint}

Confining our attention to two-body channels, the unitarity of the $S$ matrix is given by

$$
\delta_{f i}=S_{f n}^{\dagger} S_{n i},
$$

and the definition of the $T$ matrix is

$$
S_{f i}=\delta_{f i}+2 i \rho_{f} T_{f i}
$$

where

$$
\rho_{n}=\delta\left(E-E_{n}\right)
$$


is the density of states near the scattering (or reaction) energy E. Substitution of Eq.(2) into Eq.(1) gives the unitary constraint of the $T$ matrix

$$
T_{f i}-T_{f i}^{\dagger}=2 i \sum_{n} T_{f n}^{\dagger} \rho_{n} T_{n i}
$$

It may be worthwhile to note that although the well known optical theorem[20]

$$
\sigma_{T}=\frac{4 \pi}{k} \operatorname{Im} f(0)
$$

follows from Eq.(4), this relation is not equivalent to the unitarity constraint. In fact, Eq.(4) provides a constraint between observables of angular distribution, which don't appear in the optical theorem, which relates the total cross section to the forward scattering amplitude.

The unitarity constraint does not uniquely determine the $T_{f i}$, but it is highly restrictive - even in the case of elastic scattering. Assuming parity and time-reversal invariance, apropos to the strong interactions, we have

$$
\operatorname{Im} T_{11}^{-1}=-\rho_{1},
$$

where channel ' 1 ' is the channel corresponding to the lowest lying threshold, and we are assuming that we are working at energies below the next threshold. This is just the famous result that the $S$ matrix lies on the unitary circle, expressed in the language of the unitary constraint on the $T$ matrix.

The fact that the unitarity constraint is highly restrictive can be seen by recognizing that neither scale nor phase transformations of a set of unitary amplitudes preserve the unitarity constraint, Eq.(4). That is, given a set of amplitudes $T_{i j}$ that satisfy Eq.(4), the transformations

$$
T_{i j} \rightarrow \alpha_{i j} T_{i j}, \quad T_{i j} \rightarrow e^{\theta_{i j}} T_{i j},
$$

(or any combintation of these) no nontrivial set of $\alpha_{i j} \in \mathbb{R}$ and $\theta_{i j} \in \mathbb{R}$ preserve Eq.(4). This is clearly a consequence of the fact that the unitarity constraint relates quantities which are linear in the matrix elements $T_{i j}$ on the 'left-hand' side of Eq.(4) to those which are quadratic on the 'right-hand' side. This observation has an important effect when analyzing multichannel scattering and reaction data. Clearly the normalization of, for example, the integrated elastic cross section (or any reaction cross section) is determined by the unitarity constraint since the integrated cross section is related to the square of the corresponding amplitude.

\subsection{The $R$ matrix formalism}

We consider only $2 \rightarrow 2$ body scattering and reaction processes for light nuclear systems. In $R$ matrix theory, configuration space is partitioned into an interior, strongly interacting region and an exterior, Coulomb or non-polarizing interaction region by giving a channel radius $a_{c}$ for each twobody channel. The boundary of separation of these regions is the channel surface, $\mathcal{S}=\sum_{c} \mathcal{S}_{c}$.

The $R$ matrix is computed as the projection on channel surface functions

$$
\left(\mathbf{r}_{c} \mid c\right)=\frac{\hbar}{\sqrt{2 \mu_{c} a_{c}}} \frac{\delta\left(r_{c}-a_{c}\right)}{r_{c}}\left[\left(\phi_{s_{1}}^{\mu_{1}} \otimes \phi_{s_{2}}^{\mu_{2}}\right)_{s}^{\mu} \otimes Y_{\ell}^{m}\left(\hat{\mathbf{r}}_{c}\right)\right]_{J}^{M}
$$

of the Green's operator, $G_{B}=\left(H+\mathcal{L}_{B}-E\right)^{-1}$

$$
R_{c^{\prime} c}=\left(c^{\prime}\left|\left(H+\mathcal{L}_{B}-E\right)^{-1}\right| c\right)=\sum_{\lambda} \frac{\left(c^{\prime} \mid \lambda\right)(\lambda \mid c)}{E_{\lambda}-E}
$$


where $\mathcal{L}_{B}$ is the Bloch operator

$$
\left.\mathcal{L}_{B}=\sum_{c} \mid c\right)\left(c \mid\left(\frac{\partial}{\partial r_{c}} r_{c}-B_{c}\right),\right.
$$

which accounts for the presence of a boundary condition, $B$ on the channel surface. The Bloch operator ensures that the operator $H+\mathcal{L}_{B}$ is a compact, Hermitian operator (for real values of the boundary condition as is the case in the Wigner-Eisenbud $R$ matrix formulation[16]) having a real, discrete spectrum. The $R$-matrix parameters $E_{\lambda}$ and $\gamma_{\lambda c}=(c \mid \lambda)$ describe the spectrum and residues of the resolvent operator; they are treated as parameters adjusted to fit the observed data. Both hadronic and electromagnetic (ie. $\gamma-{ }^{9} \mathrm{~B}$ ) channels can be handled in this approach. The transition matrix, $\mathbf{T}$ the square of which gives the observables (cross section, etc.) of the theory, is given in matrix notation (bold type) as

$$
\mathbf{T}=\rho^{1 / 2} \mathbf{O}^{-1} \mathbf{R}_{L} \mathbf{O}^{-1} \rho^{1 / 2}-\mathbf{F} \mathbf{O}^{-1}
$$

where $\mathbf{R}_{L}=\left(\mathbf{R}^{-1}-\mathbf{L}+\mathbf{B}\right)^{-1}, \mathbf{L}=\rho \mathbf{O}^{\prime} \mathbf{O}^{-1}$, and $\mathbf{F}=\operatorname{Im} \mathbf{O}$, where $\mathbf{O}$ is the diagonal matrix of outgoing (Coulomb) wave functions in the exterior region.

The $R$-matrix approach is implemented in the EDA (Energy Dependent Analysis) code developed by Hale and collaborators[17]. The available two-body scattering and reaction data is described by minimization of the $\chi^{2}$ function with respect to variation of the $R$-matrix parameters $E_{\lambda}$ and $\gamma_{\lambda c}$, truncated to a finite number of terms in Eq.(9).

\section{Analysis and results}

We present preliminary results for multichannel unitary analyses of several compound nuclear systems.

The $R$-matrix configuration for each analysis, constructed for input into the EDA code, is given in terms of the included channel partitions (pairs), the $L S$ terms for each partition, and the channel radii and boundary conditions $B_{c}$ for each channel.

\section{1 ${ }^{9} \mathrm{~B}$ four-channel analysis}

We have included in the analysis the hadronic channels: $\mathrm{d}-{ }^{7} \mathrm{Be}$ partition with threshold of $16.5 \mathrm{MeV}$ with up to $D$-waves, ${ }^{3} \mathrm{He}-{ }^{6} \mathrm{Li}$ at $16.6 \mathrm{MeV}$ up to $P$-waves, and $\mathrm{p}-{ }^{8} \mathrm{Be}^{*}$ at $16.7 \mathrm{MeV}$ up to $P$-waves. The channel radii were constrained to lie in the range between $5.5 \mathrm{fm}$ and $7.5 \mathrm{fm}$ for the particle channels. The electromagnetic $\gamma-{ }^{9} \mathrm{~B}$ channels included were $E_{1}^{3 / 2}, M_{1}^{5 / 2}, M_{1}^{3 / 2}, M_{1}^{1 / 2}, E_{1}^{5 / 2}$, and $E_{1}^{1 / 2}$ with a channel radius of $50.0 \mathrm{fm}$. The large value of the channel radius in the electromagnetic channels provides a better description of the data than for smaller values and can be heuristically understood by considering that the photon cannot be localized.

The ${ }^{9} \mathrm{~B}$ analysis is based upon data gathered from the literature and stored in the EXFOR/CSISRS database[21]. We include elastic differential cross section data for the ${ }^{6} \mathrm{Li}-{ }^{3} \mathrm{He}$ channel given in the range of ${ }^{3} \mathrm{He}$ lab energy $1.30 \mathrm{MeV}<E\left({ }^{3} \mathrm{He}\right)<1.97 \mathrm{MeV}$ [22]; integrated cross section data for ${ }^{6} \mathrm{Li}\left({ }^{3} \mathrm{He}, \mathrm{p}\right){ }^{8} \mathrm{Be}^{*}[23]$ where the final state channel is an average of the excited-states of the quasi-twobody final state of $\mathrm{p}-{ }^{8} \mathrm{Be}^{*}$ given in the range $0.66 \mathrm{MeV}<E\left({ }^{3} \mathrm{He}\right)<5.00 \mathrm{MeV}$; integrated cross section for the ${ }^{6} \mathrm{Li}\left({ }^{3} \mathrm{He}, \mathrm{d}\right){ }^{7} \mathrm{Be}[24]$ in the range $0.42 \mathrm{MeV}<E\left({ }^{3} \mathrm{He}\right)<4.94 \mathrm{MeV}$; and capture data from the ${ }^{6} \mathrm{Li}-{ }^{3} \mathrm{He}$ initial state in the energy range $0.7 \mathrm{MeV}<E\left({ }^{3} \mathrm{He}\right)<0.825 \mathrm{MeV}[25]$. 
Table 2. The resonance structure determined in the present 4-channel fit to data as described in the text. The table displays the pole location along with $J^{\pi}$ and pole-strength information, as described in the text.

\begin{tabular}{rcrrrrl}
$E_{x}(\mathrm{MeV})$ & $J^{\pi}$ & \multicolumn{1}{c}{$\Gamma(\mathrm{keV})$} & $\mathrm{Re} E_{0}(\mathrm{MeV})$ & $E\left({ }^{3} \mathrm{He}\right)(\mathrm{MeV})$ & Strength & \\
\hline 16.4754 & $1 / 2^{-}$ & 768.46 & -.1369 & -0.2054 & 0.06 & weak \\
17.1132 & $1 / 2^{-}$ & 0.14 & 0.5109 & 0.7664 & 1.00 & strong \\
17.2012 & $5 / 2^{-}$ & 871.63 & 0.5989 & 0.8984 & 0.40 & weak \\
17.2809 & $3 / 2^{-}$ & 147.78 & 0.6785 & 1.0178 & 0.77 & strong \\
17.6754 & $5 / 2^{+}$ & 33.33 & 1.0631 & 1.5947 & 0.98 & strong \\
17.8462 & $7 / 2^{+}$ & 2036.21 & 1.2339 & 1.8509 & 0.15 & weak \\
17.8577 & $3 / 2^{-}$ & 42.52 & 1.2454 & 1.8681 & 0.97 & strong \\
18.0582 & $3 / 2^{+}$ & 767.11 & 1.4459 & 2.1689 & 0.54 & weak \\
18.4229 & $1 / 2^{+}$ & 5446.32 & 1.8206 & 2.7309 & 0.03 & weak \\
18.6872 & $1 / 2^{-}$ & 10278.41 & 2.0749 & 3.1124 & 0.15 & weak \\
19.6192 & $3 / 2^{-}$ & 1478.22 & 3.0069 & 4.5104 & 0.52 & weak \\
\hline
\end{tabular}

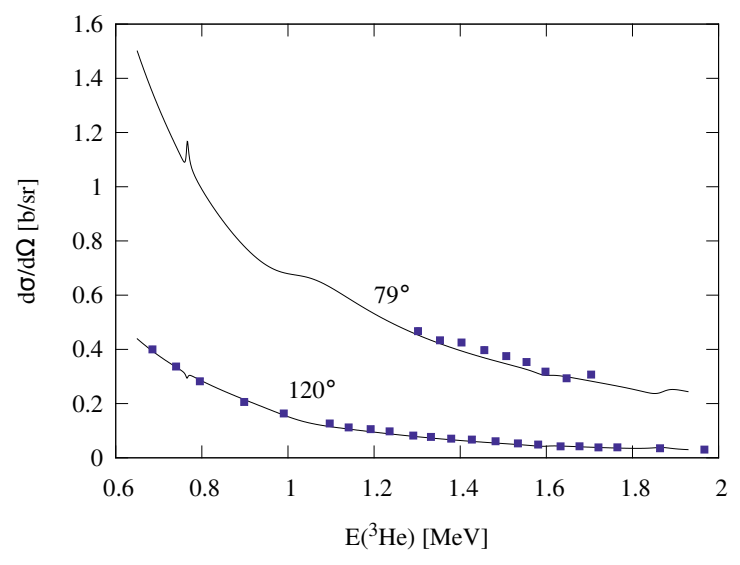

Figure 1. Comparison of elastic ${ }^{6} \mathrm{Li}\left({ }^{3} \mathrm{He},{ }^{3} \mathrm{He}\right){ }^{6} \mathrm{Li}$ differential cross section scattering data from [22] plotted against the $R$-matrix fit (solid curve) for center-of-mass differential cross section vs. ${ }^{3} \mathrm{He}$ lab energy.

Using about 40 parameters, the results of the $\chi^{2}$ minimization result in a $T$ matrix which gives the solid curves appearing in Figs.1-4, plotted along with the data obtained from references cited in the paragraphs above. The fit quality is fair, with $\chi^{2}$ /datum of 1.91, 0.55, 2.38, and 0.37 for Figs.1-4, respectively. The fit to the capture data in Fig. 4 has been folded with a Gaussian acceptance function whose width is $5 \mathrm{keV}$ to match the quoted energy resolution in Ref.[25].

The present $R$-matrix parametrization gives the resonance structure presented in Table 2 . The resonance poles of the $T$ matrix are determined by diagonalization of the complex "energy-level" matrix

$$
\mathcal{E}_{\lambda^{\prime} \lambda}(E)=E_{\lambda} \delta_{\lambda^{\prime} \lambda}-\sum_{c} \gamma_{c \lambda^{\prime}}\left[L_{c}(E)-B_{c}\right] \gamma_{c \lambda}
$$


$\mathrm{CNR} * 13$

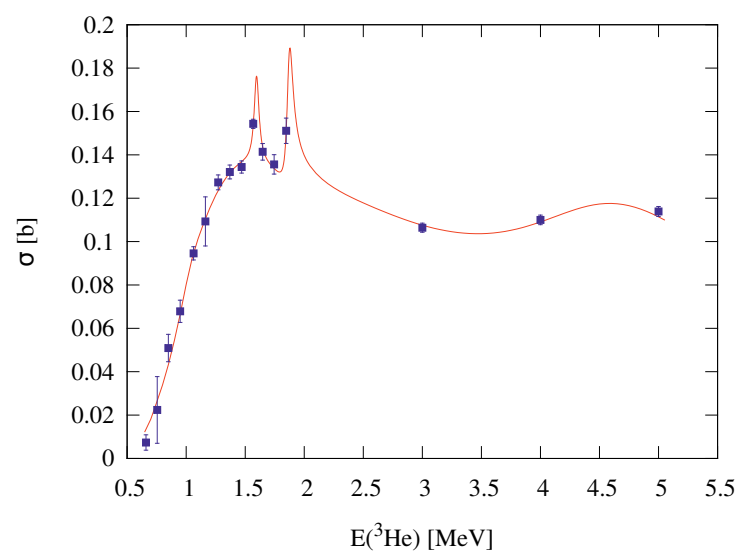

Figure 2. Comparison of the integrated cross section data for the reaction ${ }^{6} \mathrm{Li}\left({ }^{3} \mathrm{He}, \mathrm{p}\right)^{8} \mathrm{Be} *$ from [23] with the $R$-matrix fit (solid curve) vs. ${ }^{3} \mathrm{He}$ lab energy.

where $L_{c}(E)=\left.r_{c}\left(\partial O / \partial r_{c}\right) O^{-1}\right|_{r_{c}=a_{c}}, O$ is the outgoing Coulomb wave function, and $B_{c}$ is the boundary condition given at the channel radius, $a_{c}$. Details are given in Ref.[26].

The first column of Table 2 gives the real part of the pole position, $E_{0}=E_{r}-i \Gamma / 2$, where $E_{0}$ is one of the eigenvalues of the energy-level matrix, Eq.(12) relative to the ground state of ${ }^{9} \mathrm{~B}$. The spinparity is given in the second column. The width $\Gamma$ is the center-of-mass width in keV. The column labeled $E\left({ }^{3} \mathrm{He}\right)$ is the corresponding lab energy. The 'Strength' function is the ratio of the sum of the channel widths (defined in Ref.[26]) divided by the total width, $\Gamma^{-1} \sum_{c} \Gamma_{c}$. Resonances labeled 'strong' are clearly seen in at least one of the figures.

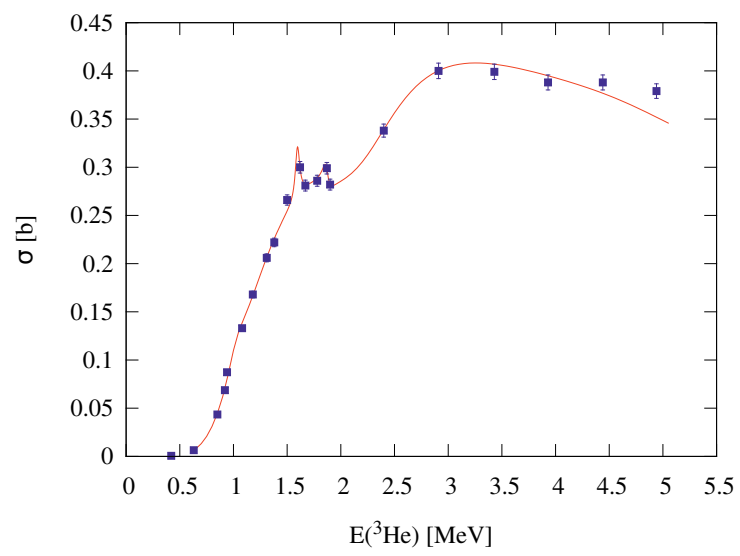

Figure 3. Comparison of the integrated cross section data for ${ }^{6} \mathrm{Li}\left({ }^{3} \mathrm{He}, \mathrm{d}\right)^{7} \mathrm{Be}$ from [24] with the $R$-matrix fit vs. ${ }^{3} \mathrm{He}$ lab energy. 


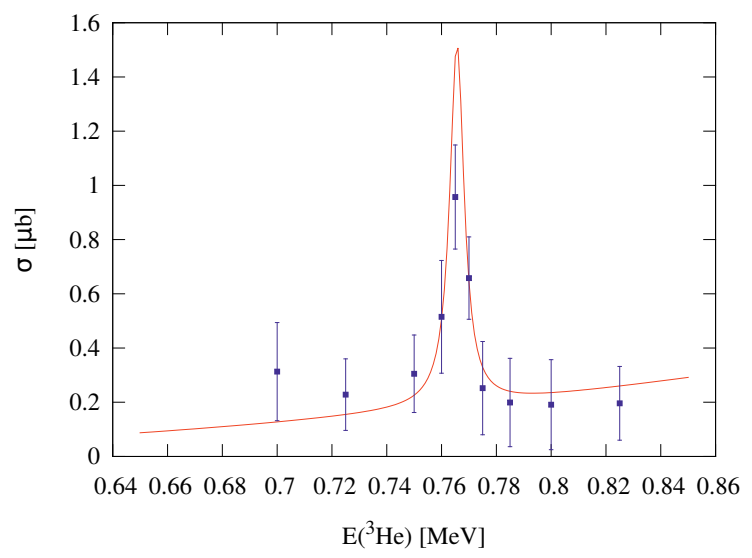

Figure 4. Comparison of capture data ${ }^{6} \mathrm{Li}\left({ }^{3} \mathrm{He}, \gamma\right){ }^{9} \mathrm{~B}$ from [25] with the $R$-matrix fit vs. ${ }^{3} \mathrm{He}$ lab energy.

The resonance structure shown in Table 2 differs significantly from that in Table 1. In particular, near the ${ }^{3} \mathrm{He}-{ }^{6} \mathrm{Li}$ and $\mathrm{d}-{ }^{7} \mathrm{Be}$ thresholds, the TUNL-NDG table lists a resonance of unknown $J^{\pi}$ at 16.024 MeV above the ${ }^{9} \mathrm{~B}$ ground state, the state considered a possible candidate in Ref.[10] for resonant destruction of ${ }^{7} \mathrm{Be}$. The current analysis has a $1 / 2^{-}$weak subthreshold resonance with a width of several hundred $\mathrm{keV}$, much too large to satisfy the desired parameters of Ref.[10] of a width of 10 's of $\mathrm{keV}$.

Possible reasons for the discrepancy include the fact that the current analysis is the first, to our knowledge, that includes much of the available data in the region below $E\left({ }^{3} \mathrm{He}\right)<3.0 \mathrm{MeV}$ in a two-body unitary analysis. Several deductions about the resonance structure in the TUNL/ENSDF tables rely on associated production of ${ }^{9} \mathrm{~B}$ experiments and single-level $R$-matrix parametrizations[12]. While more data, particularly polarization observables, would constrain the current fit with greater confidence, the present analysis appears to be the most comprehensive available that accounts for the available data in a two-body unitary way.

The resonance structure supposed in Refs.[10] and [11] for the ${ }^{9} \mathrm{~B}$ compound system are not supported by the current analysis. These works require a narrow resonance, a few 10's of keV in width within $100 \mathrm{keV}$ of the ${ }^{3} \mathrm{He}-{ }^{6} \mathrm{Li}$ (that is, $200 \mathrm{keV}$ within the $\mathrm{d}-{ }^{7} \mathrm{Be}$ ) threshold in order to explain the overproduction of ${ }^{7} \mathrm{Li}$ in $\mathrm{BBN}$ reaction network codes[7].

The current study does not conclusively eliminate the possibility of the mechanism of resonant enhancement of mass-7 destruction. The ${ }^{9} \mathrm{~B}$ compound system was identified originally by Cyburt and Pospelov[10] as playing a potential role in the destruction of ${ }^{7} \mathrm{Be}$ precisely because there is not much data in the region near the $\mathrm{d}^{7}{ }^{7} \mathrm{Be}$ threshold. Our analysis is performed on essentially the same data that the existent TUNL-NDG analyses[12] were performed, with the smallest energy probed about $400-500 \mathrm{keV}$ above the ${ }^{3} \mathrm{He}-{ }^{6} \mathrm{Li}$ threshold. It might, therefore, be suspected that the present data set would give no indication of such a low-lying resonance. Our experience with $R$ matrix analysis indicates, however, that a resonance of 10's of keV in width would likely - but not certainly - have contributions 'in the tail' to the observables considered in the present study, particularly in the ${ }^{6} \mathrm{Li}\left({ }^{3} \mathrm{He}, \mathrm{d}\right){ }^{7} \mathrm{Be}$ integrated cross section of Fig. 3 . 


\section{$\mathrm{CNR} * 13$}
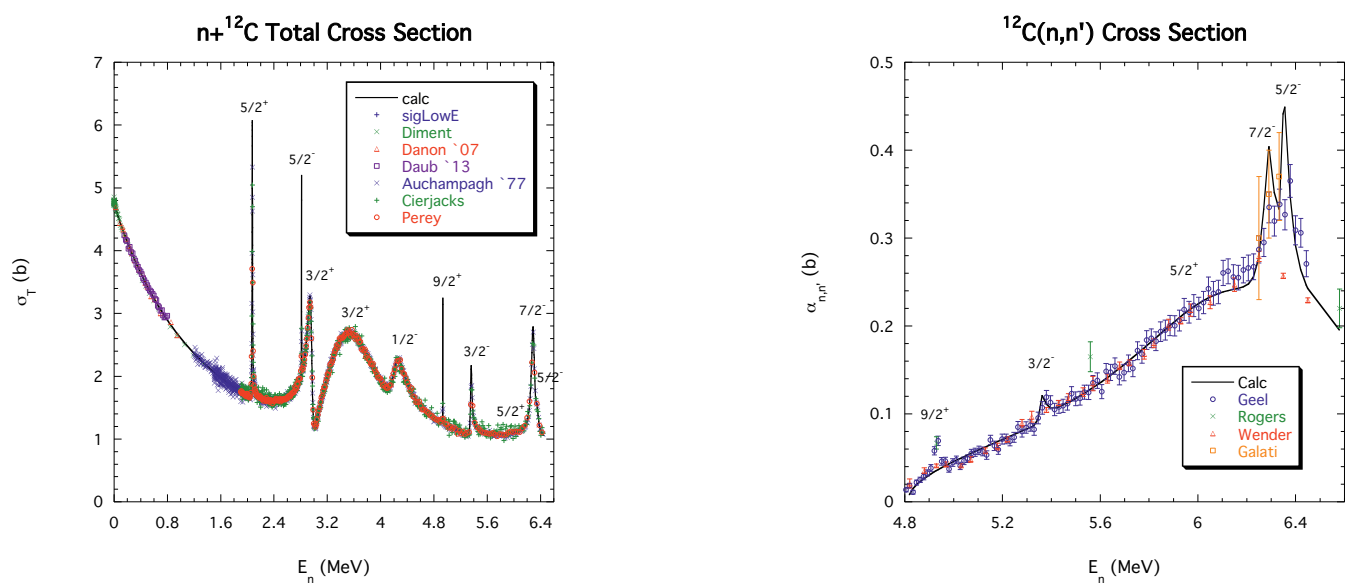

Figure 5. (Left) Total cross section, $n-{ }^{12} \mathrm{C}$ for energies $0<E_{n}<6.5 \mathrm{MeV}$. (Right) Inelastic integrated scattering cross section, ${ }^{12} C\left(n, n_{1}\right){ }^{12} C^{*}$ for energies $4.8<E_{n}<6.5 \mathrm{MeV}$.

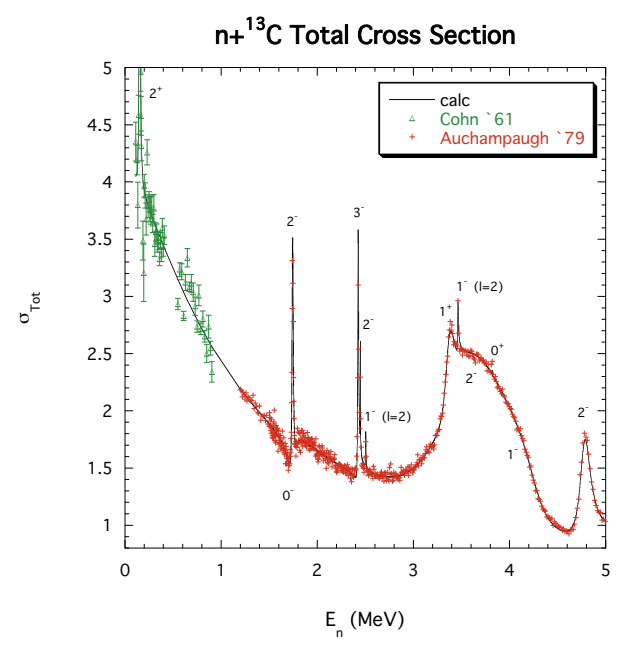

Figure 6. Total cross section, $n-{ }^{13} \mathrm{C}$ for energies $4.8<E_{n}<5 \mathrm{MeV}$. 


\begin{tabular}{|c|c|c|c|}
\hline & Channel a & $a_{c}(\mathrm{fm})$ & $I_{\max }$ \\
\hline & $n+160$ & 4.3 & 4 \\
\hline & $\alpha+{ }^{13} \mathrm{C}$ & 5.4 & 5 \\
\hline Reaction & $\begin{array}{c}\text { Energies } \\
(\mathrm{MeV})\end{array}$ & $\begin{array}{l}\text { \# data } \\
\text { points }\end{array}$ & Data types \\
\hline${ }^{16} \mathrm{O}(\mathrm{n}, \mathrm{n})^{16} \mathrm{O}$ & $E_{n}=0-7$ & 2718 & $\sigma_{T}, \sigma(\theta), P_{n}(\theta)$ \\
\hline${ }^{16} \mathrm{O}(\mathrm{n}, \alpha){ }^{13} \mathrm{C}$ & $E_{n}=2.35-5$ & 850 & $\sigma_{\text {int }}, \sigma(\theta), A_{n}(\theta)$ \\
\hline${ }^{13} \mathrm{C}(\alpha, n){ }^{16} \mathrm{O}$ & $E_{\alpha}=0-5.4$ & 874 & $\sigma_{\text {int }}$ \\
\hline${ }^{13} \mathrm{C}(\alpha, \alpha){ }^{13} \mathrm{C}$ & $E_{\alpha}=2-5.7$ & 1296 & $\sigma(\theta)$ \\
\hline total & & 5738 & 8 \\
\hline
\end{tabular}

Figure 7. $R$ matrix analysis configuration (top) and observed data (bottom) included in the analysis of the ${ }^{17} \mathrm{O}$ system.

\section{$3.2{ }^{13,14}$ C system analyses}

We present preliminary results for low energy fits to the compound systems ${ }^{13,14} \mathrm{C}$ in Figs. 5 and 6 . The independent analysis of the ${ }^{13} \mathrm{C}$ and ${ }^{14} \mathrm{C}$ systems supersedes the previous analysis of natural carbon, which is important in an array of applied contexts, including nuclear criticality safety. New data has been added to the ${ }^{13} \mathrm{C}$ system analysis, including the inelastic scattering cross section $\left(n, n_{1}\right)$ [27].

\section{$3.3{ }^{17} \mathrm{O}$ system analysis}

As an example of the importance of unitarity in parametrizations of light nuclear systems, we consider the analysis of the ${ }^{17} \mathrm{O}$ system. The configuration for the $R$ matrix analysis is given in the table in Fig.7. The table shows that this is a two-channel analysis, $n+{ }^{16} \mathrm{O}$ and $\alpha-{ }^{13} \mathrm{C}$, with unpolarized and polarized elastic $n+{ }^{16} \mathrm{O}$ data and unpolarized $\alpha+{ }^{13} \mathrm{C}$ elastic and reaction data.

Current attention is focused on discrepancies in the integrated cross sections measured earlier, in Ref.[28] and more recently by Ref.[29], shown in Fig.8.

As these figures and Fig.9 demonstrate, the data of Ref.[29] is lower by an overall factor of about $2 / 3$ from that of Ref.[28] as well as some differences in the shape of the integrated cross section. Two independent multichannel $R$ matrix analyses, one using the LANL-EDA code and the other, that of Ref.[30], have demonstrated that it is not possible to obtain a high fidelity fit of the integrated cross section of Ref.[29] without allowing the experimentally determined normalization to change. More recently, the $(\alpha, n)$ reaction was measured at low energies in Karlsruhe[31] and this data appears to agree substantially with that of Ref.[28]. The authors of Ref.[31] point out that the neutron efficiency for the Bochum measurement[29] is extrapolated from lower neutron-energy measurements of ${ }^{252} \mathrm{Cf}$ fission neutron spectra. They conclude: "Therefore, systematic deviations cannot be excluded for the data of Harissopulos et al. [29], in contrast to the present work, where the neutron efficiency was experimentally verified over a range of well-defined energies." Guided by these observations and the fact that two independent multichannel analyses of the integrated reaction cross section and elastic scattering data preclude high fidelity fits to the data of Ref.[29], we might be tempted to conclude that the older data of Ref.[28] is preferred. The matter, however, remains open. 


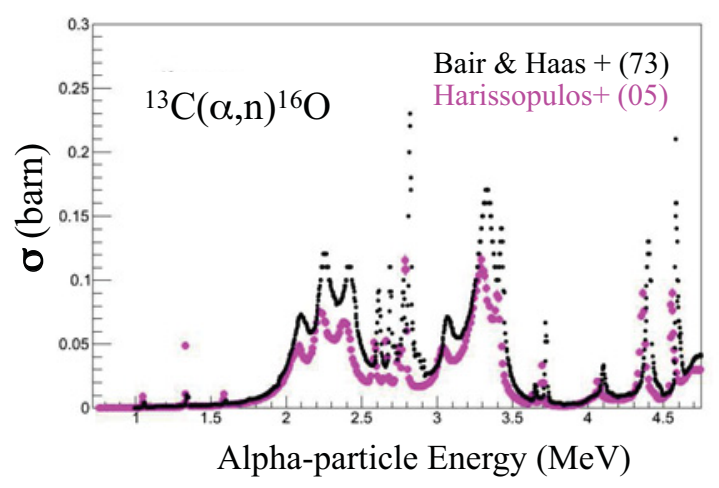

Figure 8. Discrepancy of integrated cross section measurements in Refs.[28] and [29]. Figure from Ref.[30].

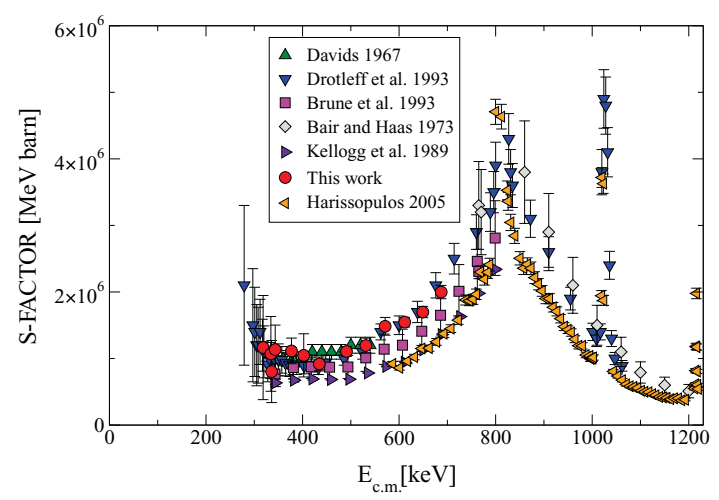

Figure 9. Comparison of various data sets, including those of Refs.[28] (diamond) and [29] (left-triangle), in terms of the astrophysical $S$ factor. Figure from Ref.[31] whose data (circles) appear to agree in scale with those of Ref.[28].

\section{Effective field theory of $d t \rightarrow n \alpha$}

In this section, we examine the reaction

$$
d+t \rightarrow n+\alpha
$$

from an effective field theory point of view. We employ modern techniques of many-body, nonrelativistic quantum field theory to describe this reaction, and also make use of the contemporary ideas of effective field theory. In the modern effective field theory approach, stable nuclei (which are treated as particles) and resonant nuclear states (which are treated as unstable particles) are described by individual fields. The fields that correspond to asymptotic states produce particles when they act on the vacuum (no-particle) state. But fields that correspond to resonances have no corresponding singleparticle states. For the reaction that we consider in this paper, we shall assume that only a single intermediate resonant state, corresponding to a spin $3 / 2^{+}{ }^{5} \mathrm{He}^{*}$, is needed. Our degrees-of-freedom 


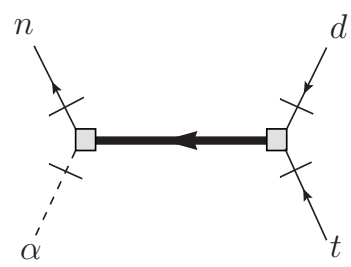

Figure 10. Graphical representation of the $d t \rightarrow n \alpha$ transition amplitude. The thick directed line is the interacting ${ }^{5} \mathrm{He}^{*}$ Green's function in the presence of the Coulomb field, $G_{*}^{(C)}(W)$. Solid, directed lines are non-zero spin particles, and the dashed line is the spin-zero $\alpha$-particle. Shaded boxes are $g_{n \alpha}$ and $g_{d t}$ couplings. The $d t$ shaded box includes the Coulomb wave function factor $\psi_{\mathbf{p}_{d t}}^{(C)}(0)$. Hash marks on the external lines indicate that they correspond to on-shell asymptotic particles, not propagators.

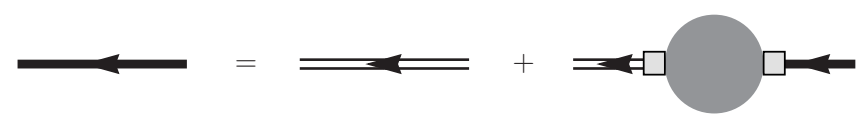

Figure 11. Diagrammatic structure of the momentum space algebraic equation for the interacting ${ }^{5} \mathrm{He}^{*}$ Green's function. The thick, directed line represents the interacting Green's function with all its self-energy corrections. The double line stands for the wrong-sign free-particle propagator. The shaded region immediately to the right of the free propagator represents the $n \alpha$ and $d t$ self energies contained in $\Sigma(W)$.

will have fields for this unstable intermediate resonance as well as those for deuteron, triton, alpha, and neutron particles.

Here we are concerned with reactions in the low-energy limit, but with a resonant intermediate state, the ${ }^{5} \mathrm{He}^{*}$ state. This introduces three parameters: two constants $g_{d t}$ and $g_{n \alpha}$ for the coupling of the $d t$ and $n \alpha$ fields to the unstable ${ }^{5} \mathrm{He}^{*}$ field, and the resonant energy of this unstable field.

A traditional method to compute coupled channel nuclear reactions is to use $R$-matrix theory. This theory entails nuclear channel radii as well as excited state energies and channel couplings. The zero channel radii limit of a two-channel, single-level $R$-matrix theory is the result:

$$
\bar{S}_{d t \rightarrow n \alpha}=\frac{8}{9} 4 \pi m_{d t} m_{n \alpha} p_{n \alpha}^{5} \frac{g_{d t}^{2}}{4 \pi} \frac{g_{n \alpha}^{2}}{4 \pi} \frac{2 \pi}{b_{0}}\left|G_{*}^{(C)}(W)\right|^{2}
$$

which is identical to the EFT result.

Figure 10 represents the $d t \rightarrow n \alpha$ fusion amplitude, the order (as also in all subsequent graphs) from right to left follows the usual convention for quantum transition amplitudes. The absolute square of this amplitude, multiplied by appropriate phase-space factors, is the fusion cross section. The ${ }^{5} \mathrm{He}^{*}$ propagator obeys the algebraic equation represented in Fig. 11, with the self-energy function denoted by the shaded region on the far right described in Fig. 12. The free-particle propagator appears with the wrong minus sign and is discussed in Ref.[32] and the subsequent companion paper, Ref.[33]. Figure 12 represents the $n \alpha$ self-energy function. The contribution of these self-energy functions appears in the absolute square of the inverse ${ }^{5} \mathrm{He}^{*}$ Green's function. 


\section{$\mathrm{CNR} * 13$}

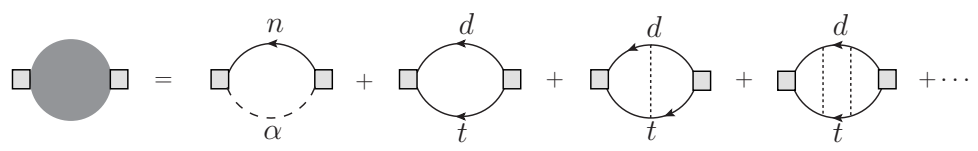

Figure 12. Self energy of the ${ }^{5} \mathrm{He}^{*}$ Green's function, including the instantaneous Coulomb interaction. The first graph stands for the $n \alpha$ self-energy function. The infinite set of terms (as indicated by the final ellipsis) include all the corrections to the $d t$ self-energy function due to instantaneous Coulomb exchanges between the charged $d$ and $t$ particles.

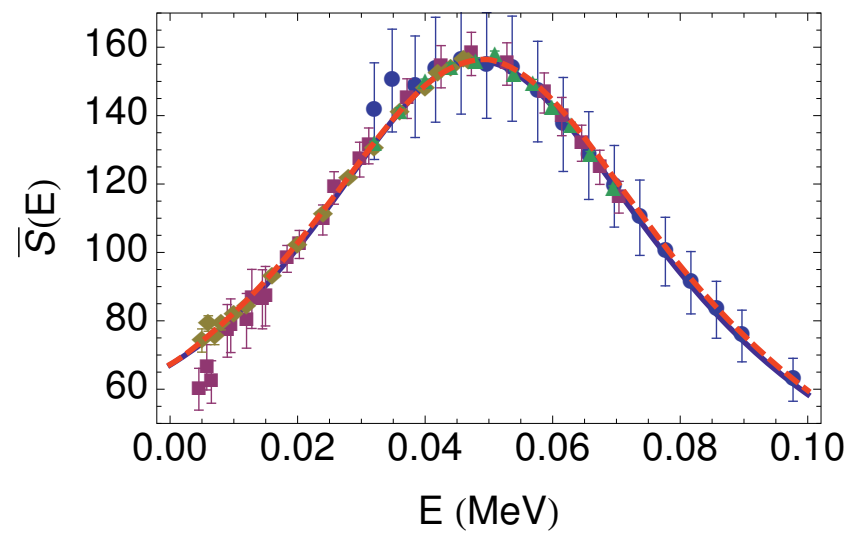

Figure 13. Dimensionless version of the astrophysical factor $\bar{S}_{d t \rightarrow n \alpha}$ for the $d t$ reaction compared with the experimental data as a function of the deuteron center-of-mass energy $E$. The solid (blue) curve is the best fit of the simple effective field theory result (14). It has a $\chi^{2}$ per degree of freedom of 0.784 . The dashed (red) curve is based on the cross section of Bosch and Hale [34] . The multilevel, multichannel $R$-matrix analysis of the ${ }^{5} \mathrm{He}$ system on which the Bosch and Hale cross sections are based includes data for $n \alpha$ and $d t$ elastic scattering, in addition to those for the associated inelastic reactions, at energies equivalent to a laboratory deuteron energy up to $11 \mathrm{MeV}$. It fits the 2665 experimental data points included using 117 free parameters with a $\chi^{2}$ per degree of freedom of 1.56. The (magenta) squares are the data of Arnold et al. [35]; the (olive) diamonds are the data of Jarmie et al. [36] renormalized by a factor of 1.017; the (green) triangles are the relative data of Brown et al. [37] renormalized by a factor of 1.025 . The necessity of these renormalizations of the experimental data is discussed in the text. The (blue) circles are the older data of Argo et al. [38] which we show for completeness but which we do not use in our fit.

A fit of this result to the data reduced to construct $\bar{S}_{d t \rightarrow n \alpha}$ is presented in Fig. 13. The fit to the $d t$ fusion cross section with our formula gives the parameter values

$$
\begin{aligned}
E_{*} & =-154 \pm 8 \mathrm{keV}, \\
\frac{g_{d t}^{2}}{4 \pi} & =199 \pm 8 \mathrm{fm}^{3} \mathrm{MeV}^{2}, \\
\frac{g_{n \alpha}^{2}}{4 \pi} & =16.4 \pm 1.0 \mathrm{fm}^{7} \mathrm{MeV}^{2} .
\end{aligned}
$$


Our result, which entails only three parameters, fits the data very well. To achieve this, it is necessary to start with a free-particle Lagrangian for the unstable ${ }^{5} \mathrm{He}^{*}$ field with the "wrong" sign. This would not be acceptable if the theory were taken to be more fundamental with an extended region of validity rather than a effective theory whose applicability is only to the low-energy regime. It is easy to show that the simple theory with two initial spin zero particles which interact via an intermediate ("s-channel") field (the simple scalar-particle analog of our theory) produces an effective range formula with a negative effective range parameter [33]. A positive effective range parameter is achieved in this theory if the intermediate field has a wrong-sign free-particle Lagrangian. Thus the restricted validity of this simple effective field theory should be acceptable just as is that of the effective range theory.

\section{Summary, findings and future work}

We have provided an overview of the light nuclear reaction program at Los Alamos, highlighting the phenomenological component of this work. We studied several ongoing analyses for varied applications including the possible resonant enhancement of the destruction of mass-7 $\left({ }^{7} \mathrm{Be}\right.$, in particular) in BBN scenarios. The near threshold, narrow state anticipated in Refs.[10] and [11] appear not to be supported by our multichannel, two-body unitary $R$-matrix analysis. We have reviewed the $R$-matrix method implemented in the Los Alamos reaction code for light nuclei, EDA and have discussed the data included from four channels: elastic ${ }^{3} \mathrm{He}-{ }^{6} \mathrm{Li},{ }^{6} \mathrm{Li}\left({ }^{3} \mathrm{He}, \mathrm{p}\right){ }^{8} \mathrm{Be} *,{ }^{6} \mathrm{Li}\left({ }^{3} \mathrm{He}, \mathrm{d}\right){ }^{7} \mathrm{Be}$ and ${ }^{6} \mathrm{Li}\left({ }^{3} \mathrm{He}, \gamma\right){ }^{9} \mathrm{~B}$.

Our analysis determines a resonance structure significantly different from that published in the TUNL-NDG/ENSDF compilation[12], as can be seen by comparing the results from the present analysis in Table 2 with the table, Table 1 for the TUNL-NDG/ENSDF analysis. Our immediate objective is to incorporate the ${ }^{8} \mathrm{Be}^{*}$ final states for each excited state (rather than average their contribution as we have done in the present analysis). This will allow the extension of the present analysis to higher energies and the incorporation of polarization data[39, 40] that we have neglected.

Our findings for the role of a putative resonance in ${ }^{9} \mathrm{~B}$ near the $\mathrm{d}-{ }^{7} \mathrm{Be}$ threshold as envisioned in Refs.[10] and [11] is that their particular mechanism of resonant enhancement of mass-7 destruction is an unlikely explanation to the ${ }^{7} \mathrm{Li}$ problem in $\mathrm{BBN}$, though low-energy data would allow a more conclusive statement of this finding or its converse.

This work was carried out under the auspices of the National Nuclear Security Administration of the U.S. Department of Energy at Los Alamos National Laboratory under Contract No. DE-AC5206NA25396 and with support from the Institute for Geophysics, Planetary Physics, and Signatures at Los Alamos National Laboratory under a University Collaborative subcontract \#257842 with the University of California at San Diego.

\section{References}

[1] Cooke, R., Pettini, M., Jorgenson, R. A., Murphy, M. T., \& Steidel, C. C. 2013, arXiv:1308.3240.

[2] Planck Collaboration, Ade, P. A. R., Aghanim, N., et al. 2013, arXiv:1303.5062.

[3] Sanders, G. H. 2013, Journal of Astrophysics and Astronomy, 34, 81.

[4] Wagoner, R. V. 1969, Astrophy. Journ. Suppl., 18, 247.

[5] Fuller, G. M., Kishimoto, C. T., \& Kusenko, A. 2011, arXiv:1110.6479.

[6] Hale, G. M., Nucl. Sci. Eng. 104, 776 (2011).

[7] B. D. Fields, Ann. Rev. Nucl. Part. Sci. 61, 47 (2011), and references therein.

[8] F. Spite and M. Spite, Astron. Astrophys. 115, 357 (1982).

[9] González Hernández, et al.Astron. Astrophys. 505, L13-L16 (2009). 


\section{$\mathrm{CNR} * 13$}

[10] R. H. Cyburt and M. Pospelov, Int. J. Mod. Phys. E 21, 1250004 (2012).

[11] N. Chakraborty, B. D. Fields and K. A. Olive, Phys. Rev. D 83, 063006 (2011).

[12] D. R. Tilley, J. H. Kelley, J. L. Godwin, D. J. Millener, J. E. Purcell, C. G. Sheu and H. R. Weller, Nucl. Phys. A 745, 155 (2004).

[13] Teichmann, T. and Wigner, E. P. Phys. Rev. 87, 123-135 (1952).

[14] P. L. Kapur and R. Peierls Proc. R. Soc. Lond. A 166 277-295 (1938).

[15] E. P. Wigner, Phys. Rev. 70, 15 (1946).

[16] E. P. Wigner and L. Eisenbud, Phys. Rev. 72, 29 (1947).

[17] G. M. Hale, Nuc. Data Sheets, 109, 2812-2816 (2008).

[18] A. M. Lane and R. G. Thomas, Rev. Mod. Phys. 30, 257 (1958).

[19] A. M. Lane and D. Robson, Phys. Rev. 151, 774 (1966).

[20] Bohr, N., Peierls, R., and Placzek, G., Nature 144, 200-201 (1939).

[21] http://www-nds.iaea.org/exfor/exfor.htm

[22] S. Buzhin'ski, et al., Izv. Rossiiskoi Akademii Nauk, Ser.Fiz. 43, 158 (1979).

[23] A. J. Elwyn, R. E. Holland, C. N. Davids, J. E. Monahan, F. P. Mooring and W. Ray, Phys. Rev. C 22, 1406 (1980).

[24] Cited in Holland et al., IEEE Trans. Nucl. Sci. NS-28, 1344 (1981).

[25] M. R. Aleksic and R. V. Popic, Fizika 10, 273-278 (1978).

[26] G. M. Hale, R. E. Brown and N. Jarmie, Phys. Rev. Lett. 59, 763 (1987).

[27] Hale, G.M., Nucl. Data Sheets, (2013) in press.

[28] J. K. Bair and F. X. Haas, Phys. Rev. C 7, 1356 (1973).

[29] S. Harissopulos, H. W. Becker, J. W. Hammer, A. Lagoyannis, C. Rolfs and F. Strieder, Phys. Rev. C 72, 062801 (2005) [nucl-ex/0509014].

[30] S. Kunieda, Nucl. Data Sheets (2013) in press

[31] M. Heil, R. Detwiler, R. E. Azuma, A. Couture, J. Daly, J. Gorres, F. Kappeler and R. Reifarth et al., Phys. Rev. C 78, 025803 (2008).

[32] L. S. Brown and G. M. Hale, arXiv:1308.0347 [nucl-th].

[33] G. M. Hale, L. S. Brown and M. W. Paris, arXiv:1308.0348 [nucl-th].

[34] H.-S. Bosch and G.M. Hale, Nucl. Fusion 32, 611 (1992).

[35] W.R. Arnold, J.A. Phillips, G.A. Saywer, E.J. Stovall, Jr., and J.L. Tuck, Phys. Rev. 93, 483 (1954).

[36] N. Jarmie, R.E. Brown, and R.A. Hardekopf, Phys. Rev.C 29, 2031 (1984).

[37] R.E. Brown, N. Jarmie, and G.M. Hale, Phys. Rev. C 35, 1999 (1987); These relative data were renormalized by a factor of 1.025 .

[38] H.V. Argo, R.F. Taschek, H.M. Agnew, A. Hemmendinger and W.T. Leland, Phys. Rev. 87, 612 (1952).

[39] Simons, Bull. Am. Phys. Soc., 11, 301 (1966).

[40] Simons, in Polarization Phenomena in Nuclear Reactions, Madison (1970), p. 597 (1971). 